\title{
RELACIÓN ENTRE LA CALIDAD DE SERVICIOS ACADÉMICOS Y LA SATISFACCIÓN EN LOS ESTUDIANTES DE LA Universidad Peruana Unión Filial TARAPOTO - 2017
}

\author{
Amado Arce Cobeñas* y Maritsa Arana Rodríguez \\ Unidad de Posgrado - Ciencias Empresariales \\ Universidad Peruana Unión, Lima - Perú \\ Recibido: 10 de marzo de 2017 Aceptado: 10 de abril de 2017
}

\begin{abstract}
Resumen
El propósito de esta investigación fue determinar el grado de relación que existe entre la calidad de servicios académicos y la satisfacción en los estudiantes de la Universidad Peruana Unión -Filial Tarapoto, 2017. El tipo de investigación fue descriptiva, explicativa, evaluativa y propositiva con un diseño de investigación no experimental, correlacional de corte transversal. Estuvo conformada por 476 estudiantes de la Facultad de Ciencias Empresariales y para obtener los resultados se utilizó dos instrumentos. Para medir la variable calidad de servicios académicos se utilizó el instrumento propuesto por Municio (2000), compuesto por 17 ítems y con una confiabilidad de alfa de Cronbach 0.918 , y para la variable satisfacción se utilizó el instrumento propuesto por Gento y Vivas (2003) compuesta por 77 items y con una confiabilidad de alfa de Cronbach 0.891 . Los resultados muestran que existe una relación directa significativa $\left(r=0.770^{* *} ; p<0,00\right)$ entre la Calidad de servicios académicos y la satisfacción educativa; es decir, cuando más alta sea la Calidad de servicios Académicos existirá más satisfacción educativa en los alumnos de la facultad de ciencias empresariales de la Universidad Peruana Unión - Filial Tarapoto, 2017.
\end{abstract}

Palabras clave: calidad de servicios, satisfacción educativa, instituciones educativas, universidades

\begin{abstract}
The purpose of this research was to determine the relationship between the quality of academic services and student satisfaction at the Universidad Peruana Unión, Campus Tarapoto, 2017. It is a is descriptive, explanatory, evaluative and proactive research, with a nonexperimental, cross-sectional correlation design. Furthermore, it consisted of 476 students from the Faculty of Business Sciences. I order to get the results, two instruments were used. To measure the quality variable of academic services, the instrument proposed by Municio (2000) composed of 17 items with a reliability of Cronbach's alpha 0.918, was used; and for the satisfaction variable, the instrument proposed by Gento and Vivas (2003) composed of 77 items with a reliability of cronbach's alpha 0.891 , was used. The results show that there is a significant direct relationship $(r=\llbracket 0.770 \rrbracket \wedge(* *) ; p<0,00)$ between the quality of academic services and educational satisfaction; that is to say, the higher the Quality of Academic services there will be more educational satisfaction in the students of the Faculty of Business Sciences at the Universidad Peruana Unión, Campus Tarapoto, 2017..
\end{abstract}

${ }^{*}$ Autor de la tesis. Email: amado.arce@upeu.edu.pe 
Palabras clave: Qualitiy of academic services, student satisfaction, educational institutions, universities 
Revista de Investigación Universitaria | Volumen 6 - Número 2, Julio - Diciembre, 2017 | DOI :

\section{Introducción}

Desde tiempos memorables la educación superior cumple con un papel fundamental: formar integralmente a la persona, dotarle de competencias para que pueda afrontar tanto la vida cotidiana y el ámbito laboral, y que tenga en cuenta que la educación superior es parte de un proceso en el cual se las personas se capacitan continuamente. Esto indica que una educación superior, acompañada de una óptima calidad, es fundamental para sostener el proceso anhelado de desarrollo económico y social en la que la sociedad está involucrada, teniendo en cuenta que vivimos en la era del conocimiento, el cambio continuo y la competitividad.

Hoy vivimos en una sociedad que en todas sus manifestaciones demandan calidad. Los países más desarrollados están experimentando un proceso de auge imparable en la valoración de la calidad; la investigación sobre la satisfacción estudiantil en cuanto a la calidad educativa universitaria ha ido tomando una mayor importancia en otros países, como vemos en los trabajos de González (2003), Rodríguez y Amaya (2005), Muñoz (2002), entre otros. En ellos se da a conocer la importancia de estudiar la relación satisfacción de los estudiantes respecto a la calidad educativa y su significado e influencia en el desarrollo de la universidad como institución educadora.

En nuestro país, actualmente se han implementado mecanismos de legislación de la calidad en la educación universitaria entre los cuales tenemos la Ley 29394 de Institutos y Escuelas de Educación Superior de 2009, la Ley 28740 del SINEACE de 2006, y el Decreto Legislativo 882 de Inversión. Con esto, el modelo de Acreditación para Programas de Estudios de Educación Superior Universitaria busca mejorar la calidad educativa de las universidades en un 100\% hasta fines de 2018, pues así todas las universidades tendrán 


\section{$(\mathrm{cc}) \mathrm{BY}$}

que ser acreditadas en todas sus carreras y eso significa que deberán cumplir con estándares de calidad, lo que da lugar a medir también la satisfacción de los estudiantes.

Dioses (2015) menciona que la evaluación de la calidad implica una preocupación de las universidades públicas y privadas por satisfacer al cliente, de esta manera mejorar no sólo los procesos si no también los resultados.

Las instituciones de educación superior universitaria están en este trayecto de calidad y deben estar conscientes y tener bien claro e interiorizado la filosofía de calidad, en la participación de todos los colaboradores de los diferentes niveles de la institución, para fomentar de esto una cultura que dé como resultado una educación de calidad y satisfacción de los estudiantes.

La Universidad Peruana Unión-Filial Tarapoto (UPeU-FT), no es ajena a esta realidad. En la actualidad se está trabajando en el proceso de acreditación de las diferentes carreras profesionales, teniendo como base el modelo de acreditación al SINEASE. De este modo, es relevante conocer la calidad de los servicios académicos y la satisfacción de los estudiantes de la UPeU - FT, a fin de establecer la relación del modelo de gestión de brechas académicas basado en una encuesta de satisfacción de los estudiantes.

\section{Método}

El tipo de investigación es descriptiva, explicativa, evaluativa y propositiva. Es descriptiva porque realizará un diagnóstico de las variables de estudio aplicando estadística descriptiva. El diseño de investigación es noexperimental, correlacional de corte transversal. Es no experimental porque no manipuló las variables de estudio y solo buscó encontrar la relación de las variables "estilos de aprendizaje" con "autoeficacia emprendedora". Asimismo, 
es correlacional por que buscó la relación entre dos variables y trasversal, porque se aplicó el instrumento una sola vez.

\subsection{Participantes}

Por otro lado, esta investigación tiene como unidad de análisis a los estudiantes de la Facultad de Ciencias Empresariales de la UPeU-FT, lo que hace una población de 476 personas.

La Facultad de Ciencias Empresariales está compuesta por las carreras: C.P Administración con Mención en Gestión Empresarial, C.P Marketing y Negocios Internacionales y C.P Contabilidad y gestión tributaria.

Tabla 1

Facultad de Ciencias Empresariales

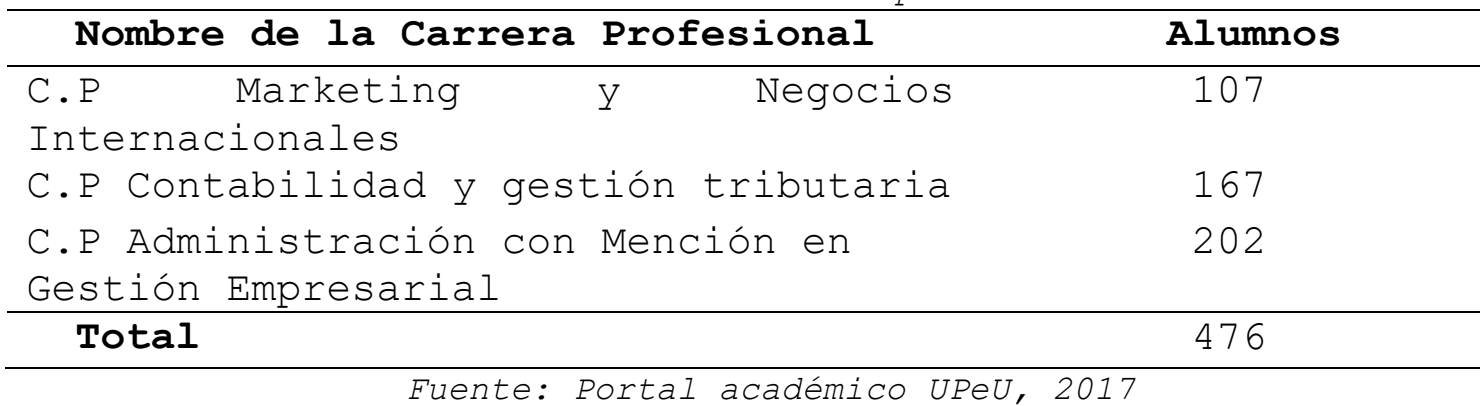

Para esta investigación se tomará el 100\% de los alumnos de la Facultad de Ciencias empresariales de la UPeU-FT, matriculados en el ciclo 2017 II, que son un total de 476 alumnos, como ya se señaló antes.

\subsection{Instrumentos}

En este trabajo se usará un instrumento de medición para la calidad de servicios académicos propuesto por Municio (2000), con escala de Likert y presenta las siguientes dimensiones: relevancia con 6 ítems, eficiencia y eficacia con 5 ítems y equidad con 6 ítems. Para medir la satisfacción de utilizará el instrumento propuesto por Gento y Vivas (2003), con escala de Likert y presenta las siguientes 
dimensiones: condiciones básicas de la infraestructura con 13 ítems, servicios de la institución con 12 ítems, condiciones de seguridad con 5 ítems, condición a su situación económica con 4 ítems, seguridad emocional por el trato afectuoso con 8 ítems, sentido de pertenencia a la Institución y al grupo de clase con 10 ítems, proceso de enseñanza y aprendizaje con 19 ítems, logros personales con 6 ítems.

\subsection{Análisis de datos}

Para el procesamiento y análisis de datos se codificó en orden correlativo el total de encuestas, luego se tabulará los datos en el software SPSS versión 20. Posteriormente se procedió a analizar los datos que generaría el sistema y por último la interpretación.

\section{Resultados}

\subsection{Resultados 1}

Tabla 2

Análisis de correlación entre la calidad de servicios educativos y la satisfacción de los alumnos de la facultad de ciencias empresariales de la Universidad Peruana Unión Filial Tarapoto 2017.

Satisfacción educativa

\begin{tabular}{|c|c|c|c|}
\hline \multirow{3}{*}{ Calidad de servicios educativos } & \\
\hline & $\begin{array}{c}\text { Rho } \\
\text { Spearman }\end{array}$ & $\begin{array}{c}\mathrm{p}- \\
\text { valor }\end{array}$ & $\mathrm{n}$ \\
\hline &, $770^{\star *}$ & .000 & 476 \\
\hline
\end{tabular}

En la Tabla 2 se presenta el análisis estadístico Rho de Spearman en el cual se obtuvo el coeficiente de 0,770 (correlación directa) y un p valor igual a 0,000 (p-valor < $0.05)$; por lo tanto, se rechaza la hipótesis nula y se acepta la hipótesis alterna. Esto quiere decir que, a mayor nivel de calidad de servicios educativos, el nivel de satisfacción 
de los estudiantes también aumentará. Esta relación indica que los estudiantes perciben que la calidad del servicio educativo es buena y por ende están satisfechos.

\subsection{Resultado 2}

Tabla 3

Análisis de correlación entre la relevancia y la satisfacción de los estudiantes.

Satisfacción de los estudiantes

Relevancia

\begin{tabular}{ccc}
\hline $\begin{array}{c}\text { Rho } \\
\text { Spearman }\end{array}$ & p-valor & $n$ \\
\hline, 590 & .000 & 476 \\
\hline
\end{tabular}

**. La correlación es significativa en el nivel, 05.

En la Tabla 3 se presenta el análisis estadístico Rho de Spearman, donde se obtuvo el coeficiente de 0,590 (correlación positiva media) y un p valor igual a 0,000 (p-valor < 0.05), por lo tanto, se rechaza la hipótesis nula y se acepta la hipótesis alterna, la relevancia se relaciona medianamente con la satisfacción de los estudiantes. Indicando que, a mayor nivel de relevancia aumentara el nivel de satisfacción de los estudiantes. Pero aún es necesario dar más participación al estudiante en el aula de clases, pues para ellos es relevante involucrarse en la enseñanza y sentir que aportan calidad. También mantener retroalimentación constante e innovar en las maneras desarrollar las clases. Esto contribuirá al fortalecimiento de la relación entre la relevancia y la satisfacción.

\section{3 Resultado 3}

Tabla 4

Análisis de correlación entre la eficiencia-eficacia y la satisfacción de los estudiantes.

Eficiencia y eficacia Satisfacción de los estudiantes 


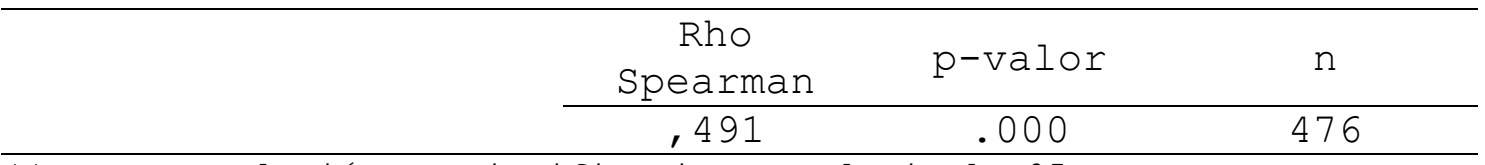

**. La correlación es significativa en el nivel, 05.

En la Tabla 4 se presenta el análisis estadístico Rho de Spearman, donde se obtuvo el coeficiente de 0,491 (correlación positiva media) y un p valor igual a 0,000 (p-valor < 0.05); por lo tanto, se rechaza la hipótesis nula y se acepta la hipótesis alterna, la eficiencia y eficacia tienen una relación moderada con la satisfacción de los estudiantes. Indicando que, a mayor nivel de eficiencia y eficacia aumentara el nivel de satisfacción de los estudiantes. Los estudiantes perciben que debe darse prioridad a la mejora de la infraestructura, y también aumentar la inversión en actividades académicas y ampliar la cobertura de la educación a través de concursos y actividades tanto internas como externas, esto permitirá aumentar el prestigio y conocimiento de la institución, por ende, fortalecer la relación entre la eficiencia y eficacia con la satisfacción.

\subsection{Resultado 4}

Tabla 4

Análisis de correlación entre la equidad y la satisfacción de los estudiantes.

Satisfacción de los estudiantes

\begin{tabular}{cccc}
\cline { 2 - 4 } Equidad & $\begin{array}{c}\text { Rho } \\
\text { Spearman }\end{array}$ & p-valor & $n$ \\
\cline { 2 - 4 } & .539 & .000 & 476 \\
\hline
\end{tabular}

**. La correlación es significativa en el nivel, 05.

En la Tabla 4 se presenta el análisis estadístico Rho de spearman. Se obtuvo el coeficiente de 0,491 (correlación positiva media) y un p valor igual a 0,000 (p-valor <0.05); por ende, se rechaza la hipótesis nula y se acepta la hipótesis alterna, la equidad tiene relación moderada con la 
satisfacción de los estudiantes. Indicando que, a mayor nivel de Equidad aumentara el nivel de satisfacción de los estudiantes. Para fortalecer la relación entre la equidad y la satisfacción, es importante mantener a la institución en un proceso de mejora continua. También comunicar a los familiares sobre los servicios educativos brindados por la institución y las facilidades que ofrece la institución para financiar los estudios; O sea mantener una relación de comunicación contante con los familiares de los estudiantes involucrándolos en las actividades académicas que se realizan.

\section{Conclusión}

Bajo la interpretación y el análisis de los resultados de este trabajo, se puede concluir señalando:

Respecto al objetivo general planteado, se encontró que existe una relación directa significativa $\left(r=0.770^{* *} ; p<\right.$ 0,00)entre la calidad de servicios académicos y la satisfacción educativa; es decir, cuando más alta sea la Calidad de servicios Académicos existirá más satisfacción educativa en los alumnos de la Facultad de Ciencias Empresariales de la UPeU-FT 2017.

Con respecto al primer objetivo específico, se encontró que existe una relación moderada $(r=\llbracket(0.590 \rrbracket \wedge(* *) ; p<0.00)$ entre la relevancia y la satisfacción de los estudiantes; donde podemos determinar que a mayor relevancia mejor serán la satisfacción de los estudiantes de la Facultad de Ciencias Empresariales de la UPeU-FT.

Con respecto al segundo objetivo específico, se encontró que existe una relación directa significativa (r= [0.491》^ $(* *) ; \mathrm{p}<0.00)$ entre la eficiencia y eficacia con la Satisfacción de los estudiantes; donde podemos determinar 
que a mayor eficiencia y eficacia mejor será la satisfacción de los estudiantes de la Facultad de Ciencias Empresariales de la UPeU-FT.

Finalmente, sobre tercer objetivo específico, se encontró que existe una relación directa significativa ( $r=$ 『0.539)^ $(* *) ; \mathrm{p}<0.00)$ entre la equidad y la satisfacción de los estudiantes; donde podemos determinar que a mayor equidad mejor sería la satisfacción de los estudiantes de la Facultad de Ciencias Empresariales de la UPeU-FT.

\section{Referencias}

Amaya (2005) Measuring Service Quality: A Re-Examination and Extension. Journal of Marketing, 56, 55-68.

Dioses, M. (2015) propuesta de sistema para mejorar la caudad del servicio académico en la facultad de medicina humana de la universidad nacional de Piura

Gento Palacios, S. Y Vivas García, M. (2003). El SEUE: Un instrumento para conocer la satisfacción de los estudiantes universitarios con su educación. Acción Pedagógica, 12(2), 16-27. Recuperado de https://dialnet.unirioja.es/servlet/articulo?codigo=2972 060

González, Z. (2000). Determinación de los elementos que condicionan la calidad de la universidad: aplicación práctica de un análisis. Revista Electrónica de Evaluación Educativa, 9 (1), 83-96. Recuperado el 10 de setiembre, 1997, de http://www.uv.es/relieve/v9n1/relievev9n1_4.htm

González-Peiteado, M., \& Pino-Juste, M., \& Penado Abilleira, M. (2017). Estudio de la satisfacción percibida por los estudiantes de la UNED con su vida universitaria. RIED. Revista Iberoamericana de Educación a Distancia, 20 (1), $243-260$.

Municio, P. (2000). Herramientas para la evaluación de la calidad.

Muñoz (2002) "Merging Service Quality and Service Satisfction. An Empirical Test of an Integrative Model." Journal of Economic Psychology, 18(4), 387-406. 